\title{
Garbage Collection for Multicore NUMA Machines
}

\author{
Sven Auhagen \\ University of Chicago \\ sauhagen@cs.uchicago.edu larsberg@cs.uchicago.edu
}

\author{
Matthew Fluet \\ Rochester Institute of \\ Technolgy \\ mtf@cs.rit.edu
}

\author{
John Reppy \\ University of Chicago \\ jhr@cs.uchicago.edu
}

\begin{abstract}
Modern high-end machines feature multiple processor packages, each of which contains multiple independent cores and integrated memory controllers connected directly to dedicated physical RAM. These packages are connected via a shared bus, creating a system with a heterogeneous memory hierarchy. Since this shared bus has less bandwidth than the sum of the links to memory, aggregate memory bandwidth is higher when parallel threads all access memory local to their processor package than when they access memory attached to a remote package. This bandwidth limitation has traditionally limited the scalability of modern functional language implementations, which seldom scale well past 8 cores, even on small benchmarks.

This work presents a garbage collector integrated with our strict, parallel functional language implementation, Manticore, and shows that it scales effectively on both a 48core AMD Opteron machine and a 32-core Intel Xeon machine.
\end{abstract}

Categories and Subject Descriptors $\quad$ D.3.0 [Programming Languages]: General; D.3.2 [Programming Languages]: Language Classifications-Concurrent, distributed, and parallel languages; D.3.4 [Programming Languages]: Processors-Memory management (garbage collection)

General Terms Languages, Performance

Keywords garbage collection, parallelism, NUMA

\section{Introduction}

Inexpensive multicore processors and accessible multiprocessor motherboards have brought all of the challenges inherent in parallel programming with large numbers of threads with non-uniform memory access (NUMA) into the

Permission to make digital or hard copies of all or part of this work for personal or classroom use is granted without fee provided that copies are not made or distributed for profit or commercial advantage and that copies bear this notice and the full citation on the first page. To copy otherwise, to republish, to post on servers or to redistribute to lists, requires prior specific permission and/or a fee.

MSPC'11, June 5, 2011, San Jose, California, USA.

Copyright (c) 2011 ACM 978-1-4503-0794-9/11/06 . . \$10.00 foreground. Functional programming languages are a particularly interesting approach to programming parallel systems, since they provide a high-level programming model that avoids many of the pitfalls of imperative parallel programming. But while functional languages may seem like a better fit for parallelism due to their ability to compute independently while avoiding race conditions and locality issues with shared memory mutation, implementing a scalable functional parallel programming language is still challenging. Since functional languages are value-oriented, their performance is highly dependent upon their memory system.

Our group has been working on the design and implementation of a parallel functional language to address the opportunity afforded by multicore processors. In this paper, we focus on the design of our memory system and parallel garbage collector. This system is designed to minimize required synchronization and to maximize locality, two features which have proven crucial to the scalability of our system on larger machines. Recent work on other functional languages has shown that the memory system is the limiting factor to improved performance for many types of code [MPS09, And10]. Our work has been guided by measurements of a number of parallel benchmarks; we present detailed results from a representative subset of these programs.

This paper makes the following contributions:

1. We demonstrate a modern functional language that makes effective use of a large number of modern NUMA multicore processors. The best recent work scales to no more than 12 cores, and we demonstrate good utilization of all available cores on both 32 and 48 core machines. This scaling is demonstrated through a set of small but representative benchmarks across a variety of physical memory allocation strategies.

2. We describe our garbage collector, which provides excellent performance on multicore, NUMA machines. While some of the individual ideas in the garbage collector build on classic work [App89, DL93, DG94], we present a novel approach that, when combined with other aspects of our runtime architecture designed to maximize locality, avoids bottlenecks due to excessive memory traffic. 
The remainder of the paper is organized as follows. In the next section, we describe our language and runtime system. Section 3 lays out the architecture of our garbage collector. Section 4 contains a detailed evaluation of our implementation using some representative benchmarks. Due to length constraints, a discussion of related work is omitted.

\section{Manticore overview}

The Manticore project encompasses both design and implementation of parallel functional programming languages on modern multicore and multiprocessor systems. In this section, we give a brief overview of the features relevant to threading and the garbage collector. More detail can be found in our previous papers $\left[\mathrm{FRR}^{+} 07, \mathrm{FFR}^{+} 07\right]$.

\subsection{Programming model}

Parallel ML (PML) is the programming language supported by the Manticore system. Our programming model is based on a strict, but mutation-free, functional language (a subset of Standard ML [MTHM97]), which is extended with support for multiple forms of parallelism. This subset includes most of the core features of SML as well as a simple module system. PML differs from SML primarily by lacking mutable data (i.e., reference cells and arrays), but it does include exceptions. PML extends this sequential core with both fine-grained implicitly-threaded and coarse-grain explicitly-threaded [RRX09] parallel-programming mechanisms. The implicitly-threaded mechanisms include a variety of lightweight syntactic forms that allow the programmer to suggest to the compiler and runtime system that parallelism would be beneficial [FRRS08]; because the threads used to evaluate these constructs are not visible at the language level, the constructs are termed implicitly threaded. The explicitly-threaded mechanisms include language-level visible threads and synchronous message passing, providing a parallel implementation of Concurrent ML's concurrency primtives [RRX09].

\subsection{The Manticore runtime system}

The Manticore runtime system consists of a hardware abstraction level, which is written in $\mathrm{C}$, that supports virtual processors (vproc), basic system services, such as I/O and networking, and a parallel garbage collector. A vproc is an abstraction of a computational resource, and is used to execute code and balance work across the system. Each vproc is hosted by its own pthread [But97], which is pinned to a physical node. When there are less vprocs than processors, they are assigned sparsely across the nodes to minimize contention on the node-shared L3 cache.

\subsection{Execution of parallel work and locality}

All of the implicitly threaded parallelism language features work by pushing units of parallel work (in the form of continuations) onto a vproc-local work queue and then beginning execution of the first unit of work. If a vproc has no work to perform, then it uses work-stealing to find a unit of pending work on another vproc and begins executing it. This strategy is designed to keep memory and computation local to the thread that began the work whenever possible and leads to one of the key invariants provided by our runtime system and used by our garbage collector — all data is local to a processor unless it was either captured in a closure and stolen by another processor or it is passed in a message by the CML explicit threading features. At these two points, the runtime and basis library handle copying data out of the local heap and into the global space, as we describe in Section 3.1. This invariant means that:

1. There are no pointers from one vproc's local heap to another's.

2. There are no pointers from the global heap into any vproc's local heap.

Many related collectors require these properties to obtain concurrency or parallelism. Our approach differs from theirs by requiring neither write barriers nor static analysis to maintain these properties.

\section{GC and heap}

Our garbage collector is based on a novel combination of the Doligez-Leroy-Gonthier (DLG) parallel collector [DL93, DG94] and the Appel semi-generational collector [App89]. This design allows us to minimize GC synchronization between vprocs and to preserve locality.

\subsection{Heap architecture}

We use the DLG heap architecture of per-vproc local heaps combined with a global heap. As in the DLG collector, we maintain the invariant that there are no pointers between local heaps or from the global heap into the local heap. This invariant means that for one vproc to communicate an object to another, we must first promote the object to the global heap. The cost of promotion can be a significant burden, so we have developed a number of techniques for reducing the amount of promoted data. These include a lazy promotion scheme for work stealing [Rai10] and the use of object proxies. ${ }^{1}$

Functional-language implementations are notorious for their high rate of memory allocation. Fortunately, most of this data is ephemeral and so generational techniques are quite effective. To this end, we use Appel's semigenerational heap architecture for the local heaps. The local heaps are fixed size that is chosen so that the local heaps will fit into the L3 cache.

The global heap is organized into a collection of chunks. Each vproc has a current chunk that it uses when it needs to allocate in or promote an object to the global heap. In a

\footnotetext{
${ }^{1}$ Object proxies are a special kind of object that is used to allow references from the global heap back into the local heap. We use them in the implementation of our explicit concurrency constructs.
} 


\section{Object length (48 bits)}

ID (15 bits) 1

Figure 1. The header word of mixed-type, raw, and vector heap objects

NUMA system, each node has its own bank of memory with the property that access from a node to its own memory is faster than access to memory on other nodes. For this reason, our memory system tracks the node on which a chunk is allocated and preserves node affinity when reusing chunks.

The main advantage of the DLG split-heap architecture is that it requires little or no synchronization between vprocs for most garbage collection activity. Our system has three different garbage-collection phases: minor, major, and global. The former two correspond to Appel's minor and major collections and are used to reclaim space in the local heap. The global collection is a parallel stop-the-world collector. We describe these in more detail below.

\subsection{Object representation and scanning}

The Manticore memory system supports three basic kinds of heap objects: raw-data objects (e.g., strings), vectors of pointers, and mixed-type objects, which contain both pointer and non-pointer data. Heap objects have a 64-bit header word as shown in Figure 1. The lowest bit is always 1, which distinguishes headers from forward pointers. The rest of the header word consists of a 15-bit ID and a 48-bit length. We reserve two IDs for raw and vector data. For mixed objects, the ID is an index into an object-descriptor table that is generated by the compiler. The object-descriptor table includes pointers to object-scanning and forwarding functions, which are also generated by the compiler.

Each garbage-collection function in the table is specifically created for the structure of the corresponding mixedtype object. This approach allows the garbage collector to avoid scanning each field of an object at runtime and instead to generate code during compilation that processes only the pointer fields of each object. We follow this approach for all mixed-type objects, though the garbage collector still distinguishes raw and vector objects and handles them directly to avoid a pointer lookup in the object table.

\subsection{Minor and major collections}

Following Appel, we divide a vproc's local heap into two separate spaces: the nursery area and the old-data area. New objects are allocated in the nursery area until it is full and a minor garbage collection is triggered. The minor garbage collector copies all live data from the nursery area to the old-data area of the local heap. After this minor garbage collection finishes, the remaining free space in the local heap is divided in half and the upper half will be used as the new nursery area. This process is illustrated in Figure 2. Because there are no pointers into the local heap from outside (other

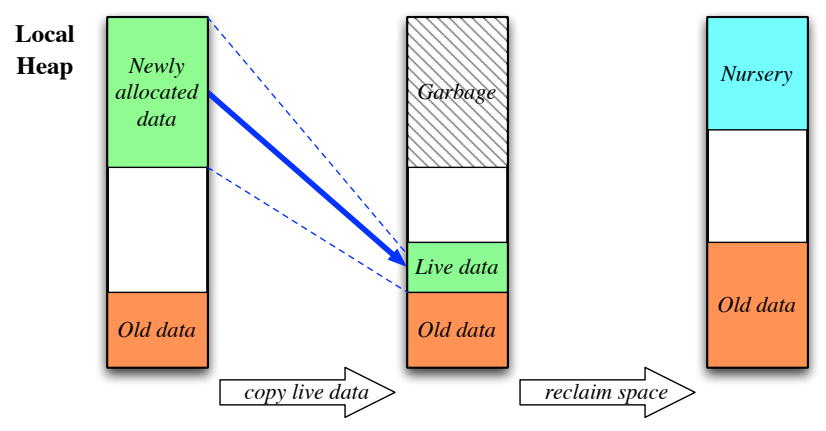

Figure 2. A minor garbage collection in Manticore

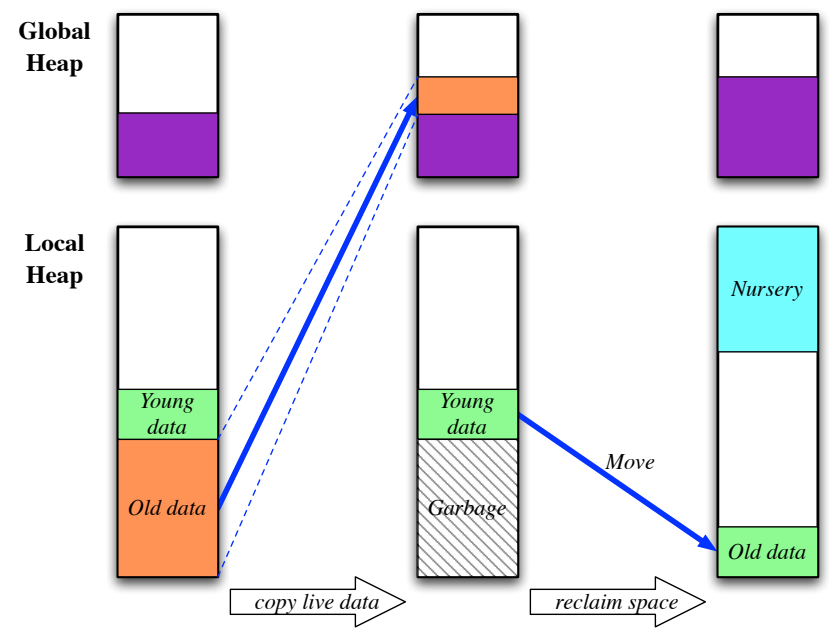

Figure 3. A major garbage collection in Manticore

than the roots), minor collections require no synchronization. A minor garbage collection triggers a major garbage collection when the size of the new nursery area falls below a certain threshold or if a global garbage collection is pending.

The major garbage collection copies the live objects from the old-data area in a vproc's local heap to its dedicated memory chunk in the global heap. To avoid premature promotion, we partition the old-data area into data that was just copied in the previous minor collection (called young data) and the data that was copied earlier. The young data are guaranteed to be live (because a minor collection always immediately precedes this major collection) and we do not copy it to the global heap. Figure 3 illustrates this process.

Major collections only require synchronization when the vproc's current memory chunk is exhausted, since, in that case, the vproc needs to allocate a new chunk of global memory. This synchronization is either node-local because it involves the reuse of a chunk of memory or global if a new chunk needs to be requested from the system and registered with the runtime. 
In addition to minor and major collections, the runtime system also implements object promotion, which is required when an object is to be shared with other vprocs. Promotion is essentially a major collection, where the root set is a pointer to the promoted object, and the synchronization requirements are the same as for major collection.

\subsection{Global collection}

Global collection is triggered when the size of global heap chunks allocated exceeds a threshold. ${ }^{2}$ The vproc that determines that a global collection first attempts to trigger a global collection. After the collection is triggered, one vproc is assigned the leadership role and performs the following actions.

1. Set a global flag that a global garbage collection is in progress and mark this vproc the leader.

2. Signal all of the other vprocs to enter garbage collection code by setting their allocation limit pointer to zero. This strategy allows the runtime to know that all vprocs will be at a safe execution point with known roots.

3. Wait for all of the other vprocs to enter the global collection, which requires first performing their parallel minor and major collections.

At this point, every vproc will be in the state shown at the end of Figure 3. Everything pointed to by the roots and local heap will be present either elsewhere in the local heap or in a global heap chunk. These global heap chunks are gathered on a per-node basis and placed into a list of from-space chunks. Each vproc then obtains a new global heap chunk and scans the vproc's roots and local heap, placing any objects pointed-to into this new to-space chunk. In parallel with one another, the vprocs obtain chunks on a per-node basis from either the from-space list or the list of to-space chunks that have not been scanned. Each of these chunks are removed and scanned until no chunks remain on the local node. Once all of the vprocs across all nodes have completed, the old from-space chunks are returned to the free-space chunk pool and execution of the program resumes.

\section{Evaluation}

Our 32 core Intel and 48 core AMD hardware is described in detail in Appendix A.

\subsection{Benchmarks}

For our empirical evaluation, we use five benchmark programs from our benchmark suite and one synthetic benchmark. Each benchmark is written in a pure, functional style and was originally written by other researchers and ported to our system. We ran each experiment 10 times and we report the average performance results in our graphs and tables.

\footnotetext{
${ }^{2}$ Currently, this threshold is the number of vprocs times $32 \mathrm{MB}$
}

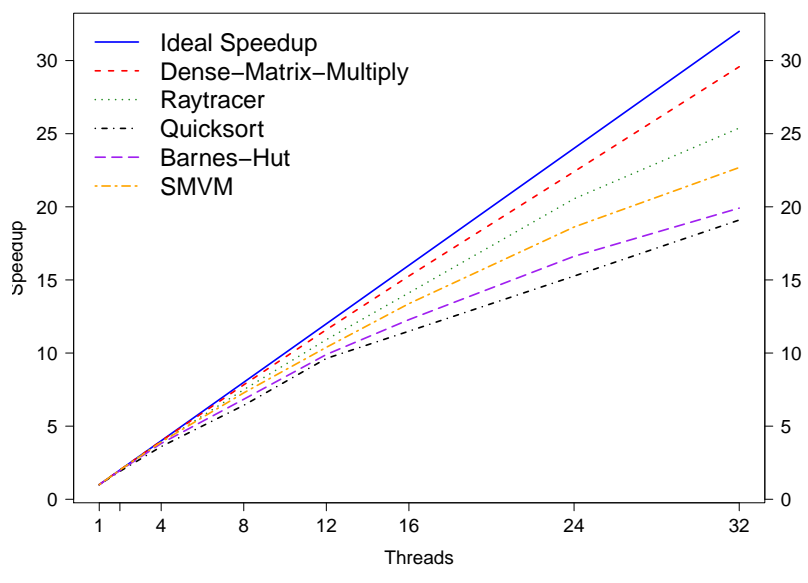

Figure 4. Comparative speedup plots for five benchmarks on Intel hardware.

The Barnes-Hut benchmark [BH86] is a classic N-body problem solver. Each iteration has two phases. In the first phase, a quadtree is constructed from a sequence of mass points. The second phase then uses this tree to accelerate the computation of the gravitational force on the bodies in the system. Our benchmark runs 20 iterations over 400,000 particles generated in a random Plummer distribution. Our version is a translation of a Haskell program [GHC].

The Raytracer benchmark renders a $512 \times 512$ image in parallel as two-dimensional sequence, which is then written to a file. The original program was written in ID [Nik91] and is a simple ray tracer that does not use any acceleration data structures. The sequential version differs from the parallel code in that it outputs each pixel to the image file as it is computed, instead of building an intermediate data structure.

The Quicksort benchmark sorts a sequence of 10,000,000 integers in parallel. This code is based on the NESL version of the algorithm [Sca].

The SMVM benchmark is a sparse-matrix by densevector multiplication. The matrix contains 1,091,362 elements and the vector 16,614 .

The DMM benchmark is a dense-matrix by dense-matrix multiplication in which each matrix is $600 \times 600$.

\subsection{Performance}

As shown in Figure 4, on the Intel machine, the dense-matrix multiplication (DMM) and raytracer benchmarks have abundant, independent parallelism and our compiler and runtime exploit them, demonstrating nearly ideal speedup over the baseline single-processor performance up to the maximum number of cores. Quicksort, barnes-hut, and sparematrix multiplication (SMVM) all see reducing speedups past 16 threads, but continue to steadily improve performance as more threads are added.

On the AMD machine, shown in Figure 5, DMM and the raytracer benchmarks perform well. But, both quicksort and barnes-hut scale nicely to 36 threads but then only take 


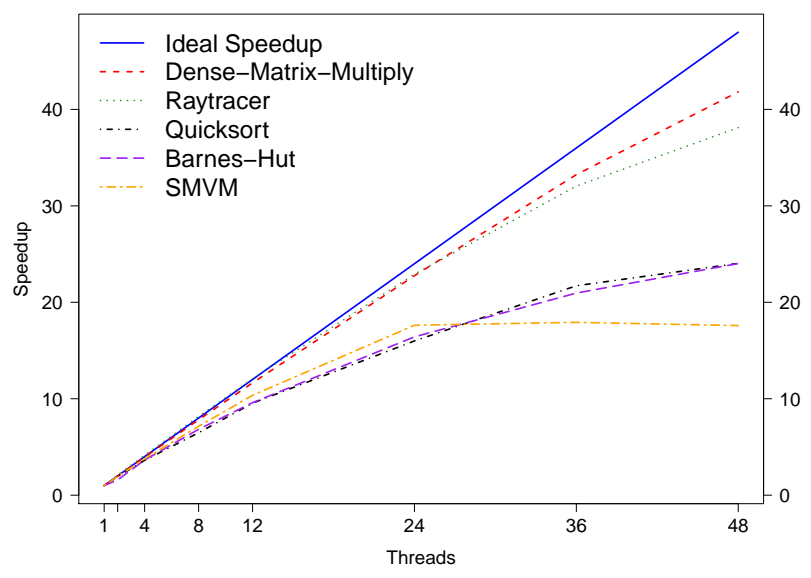

Figure 5. Comparative speedup plots for five benchmarks on AMD hardware using local memory allocation.

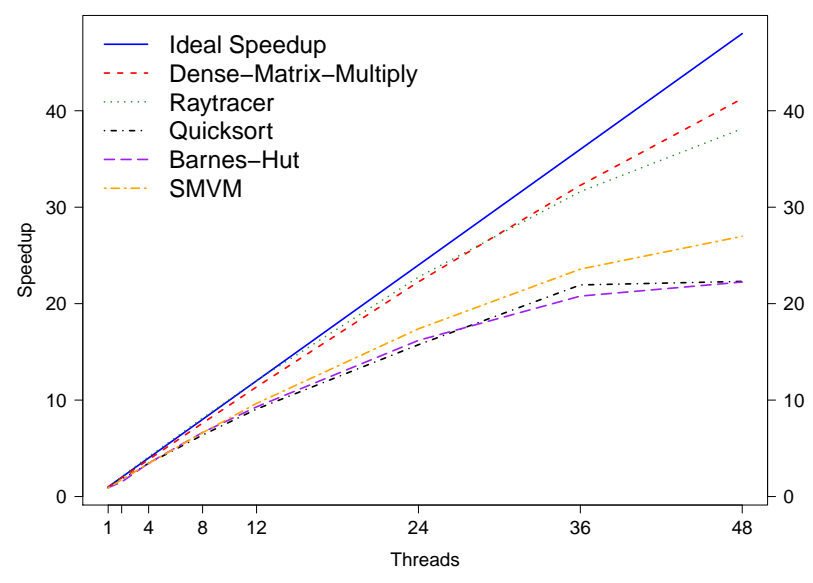

Figure 6. Comparative speedup plots for five benchmarks on AMD hardware with interleaved memory allocation.

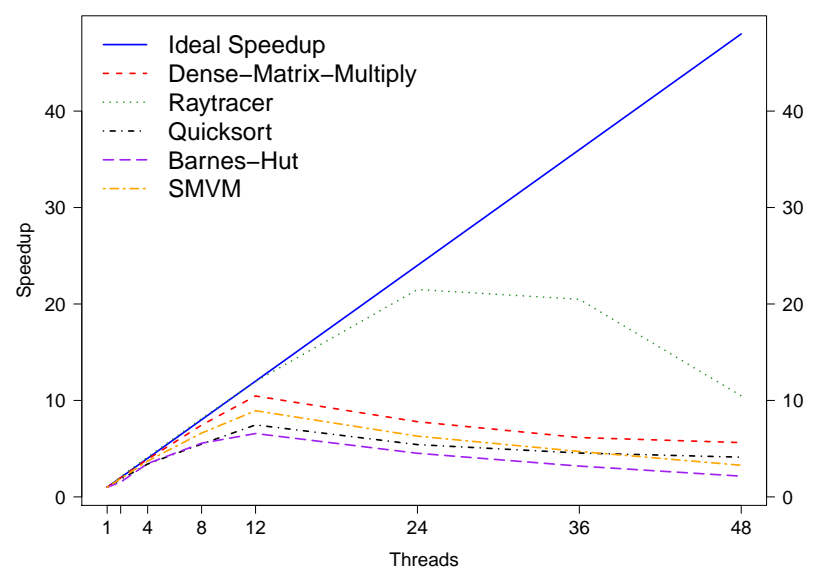

Figure 7. Comparative speedup plots for five benchmarks on AMD hardware with socket zero memory allocation. slight advantage of additional threads. In barnes-hut, we believe that this behavior is due to the sequential portion. Quicksort also is limited by its fork-join parallelism, and without significantly increasing the size of the underlying dataset, it is difficult to take advantage of the additional available parallelism.

Sparse-matrix multiplication provides the least scalability for the AMD system. We believe that this is due to a large amount of available execution parallelism but a relatively small amount of data. Unless this data is either perfectly divided between the nodes or replicated to each location, this benchmark fails to take much advantage of greater than even 24 threads. We believe that the Intel machine's greater performance, particularly on SMVM, is due to a smaller NUMA penalty when accessing the relatively smaller amount of shared data, much of which resides on only one node. Additionally, with only four nodes on the Intel machine, threads are twice as likely to be located near data even if that data was placed randomly.

Benchmarks such as dense-matrix multiplication and raytracer, with excellent locality and almost no shared data can scale nearly perfectly if all of their data is kept locally. The other benchmarks, which feature either heavily shared data or significant points that sequentially merge data before creating more parallel work show diminished improvements. In all cases, poor locality negatively affects performance, particularly on machines with multiple processor packages and relatively large numbers of cores - in our experience, between 24 and 36.

\subsection{Effect of allocation location}

By default, we allocate memory pages on the same node as the pinned vproc that required additional memory. As a further test of locality, we modified the allocator for our garbage collector with two alternative strategies that are similar to those of other functional language single-threaded and parallel garbage collectors. In Figure 6, we use an allocation strategy that balances physical page assignments between the hardware packages. This strategy is currently used in the Glasgow Haskell Compiler (GHC). In Figure 7, the allocation strategy defaults to a single node for all allocations, which is the default NUMA behavior encountered by singlethreaded garbage collectors. These speedup graphs are both plotted relative to the single-processor performance for the AMD machine in Figure 5.

Our strategy, which allocates pages local to the pinned vproc that requests and used the data, provides slightly better absolute performance at all processor counts on all benchmarks except for SMVM in the interleaved strategy at greater than 24 cores. In that benchmark, there is a small portion of data (the vector) that is accessed by all of the threads. Our default implementation encounters bus saturation on the AMD machine at larger numbers of processors, as all nodes are attempting to access data located in the same package. 
The single-node allocation strategy shows reasonable scalability until 12 cores. But, this strategy fails after that point, and we expect all collectors using this approach to require NUMA allocation tuning. ${ }^{3}$

\section{Conclusion}

We have demonstrated a garbage collector designed to make effective use of the memory hierarchy and that scales very well on a large number of processor cores. Keys to this design are private minor heaps that are collected concurrently with program execution and in parallel with one another and a major heap architecture that allows parallel collections while avoiding increasing traffic on the memory bus. Though some aspects of our system would need to be enhanced, for example with write barriers or static analysis, in the context of systems that permit and encourage frequent unrestricted memory mutation, we believe that these techniques are readily applicable to other runtimes.

Acknowledgments Thanks to Bradford Beckmann for reviewing the breakdown of the AMD G34 socket. This material is based upon work supported by the National Science Foundation under Grants CCF-0811389 and CCF-1010568. The views and conclusions contained herein are those of the authors and should not be interpreted as necessarily representing the official policies or endorsements, either expressed or implied, of these organizations or the U.S. Government.

Access to the Intel machine was provided by Intel Research. Thanks to the management, staff, and facilities of the Intel Manycore Testing Lab. ${ }^{4}$

\section{References}

[And10] Anderson, T. A. Optimizations in a private nurserybased garbage collector. In ISMM '10, Toronto, Ontario, Canada, 2010. ACM, pp. 21-30.

[App89] Appel, A. W. Simple generational garbage collection and fast allocation. $S P \& E$, 19(2), 1989, pp. 171-183.

[BH86] Barnes, J. and P. Hut. A hierarchical $O(N \log N)$ force calculation algorithm. Nature, 324, December 1986, pp. 446-449.

[But97] Butenhof, D. R. Programming with POSIX Threads. Addison-Wesley, Reading, MA, 1997.

$$
\begin{aligned}
& \text { [Car] Carver, T. Magny-cours and direct } \\
& \text { connect architecture 2.0. Available } \\
& \text { from } \\
& \text { documentation/articles/pages/ } \\
& \text { Magny-Cours-Direct-Connect-Architecture-2. } \\
& \text { 0.aspx. }
\end{aligned}
$$

\footnotetext{
${ }^{3}$ The current garbage collector for the Glasgow Haskell Compiler (GHC) recently required exactly this change in order to scale to even 7 processors across two sockets.

${ }^{4}$ Manycore Testing Lab Home:

http://www. intel.com/software/manycoretestinglab Intel Software Network:

http://www.intel.com/software
}

$\left[\mathrm{CKD}^{+}\right.$10] Conway, P., N. Kalyanasundharam, G. Donley, K. Lepak, and B. Hughes. Cache hierarchy and memory subsystem of the AMD Opteron processor. Micro, 30, 2010, pp. 16-29.

[DG94] Doligez, D. and G. Gonthier. Portable, unobtrusive garbage collection for multiprocessor systems. In POPL '94, Portland, Oregon, United States, January 1994. ACM, pp. 70-83.

[DL93] Doligez, D. and X. Leroy. A concurrent, generational garbage collector for a multithreaded implementation of ML. In POPL '93, Charleston, South Carolina, United States, January 1993. ACM, pp. 113-123.

$\left[\mathrm{FFR}^{+}\right.$07] Fluet, M., N. Ford, M. Rainey, J. Reppy, A. Shaw, and Y. Xiao. Status Report: The Manticore Project. In $M L$ '07. ACM, October 2007, pp. 15-24.

$\left[\mathrm{FRR}^{+}\right.$07] Fluet, M., M. Rainey, J. Reppy, A. Shaw, and Y. Xiao. Manticore: A heterogeneous parallel language. In DAMP '07. ACM, January 2007, pp. 37-44.

[FRRS08] Fluet, M., M. Rainey, J. Reppy, and A. Shaw. Implicitly-threaded parallelism in Manticore. In ICFP '08, Victoria, BC, Candada, September 2008. ACM, pp. 119-130.

[GHC] GHC. Barnes Hut benchmark written in Haskell. Available from http://darcs.haskell.org/ packages/ndp/examples/barnesHut/.

[Int] Intel. Intel Xeon Processor X7560. Specifications at http://ark. intel.com/Product. aspx? id $=46499$.

[MPS09] Marlow, S., S. Peyton Jones, and S. Singh. Runtime support for multicore Haskell. In ICFP '09. ACM, August-September 2009, pp. 65-77.

[MTHM97] Milner, R., M. Tofte, R. Harper, and D. MacQueen. The Definition of Standard ML (Revised). The MIT Press, Cambridge, MA, 1997.

[Nik91] Nikhil, R. S. ID Language Reference Manual. Laboratory for Computer Science, MIT, Cambridge, MA, July 1991.

[QSS] QSSC. QSSC-S4R Technical Product Specification. Available from http://www.qsscit.com/ language_config/down.php?hDFile= S4R_TPS_1.0.pdf.

[Rai10] Rainey, M. Effective Scheduling Techniques for HighLevel Parallel Programming Languages. Ph.D. dissertation, University of Chicago, August 2010. Available from http://manticore.cs.uchicago. edu.

[RRX09] Reppy, J., C. Russo, and Y. Xiao. Parallel Concurrent ML. In ICFP '09, Edinburgh, Scotland, UK, AugustSeptember 2009. ACM, pp. 257-268.

[Sca] Scandal Project. A library of parallel algorithms written NESL. Available from http: / / www . cs. cmu . edu/ scandal/nesl/algorithms.html. 


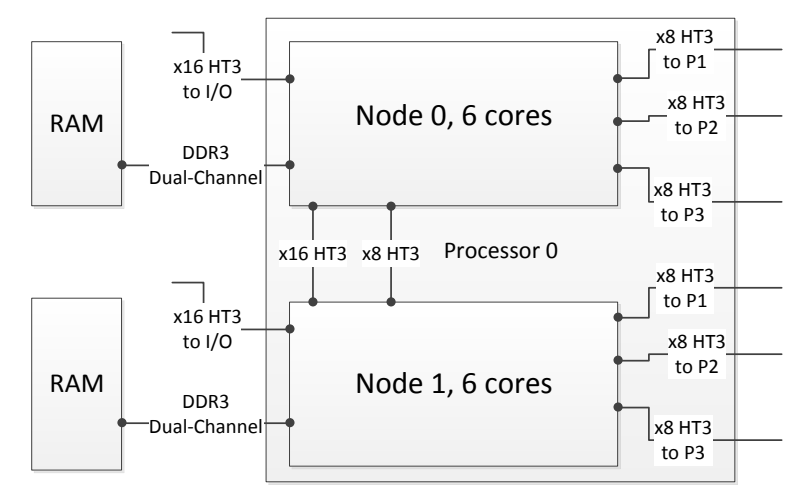

Figure 8. Interconnects for one processor in a quad AMD Opteron machine.

\section{A. Hardware}

\section{A.1 AMD Hardware}

Our AMD benchmark machine is a Dell PowerEdge R815 server, outfitted with 48 cores and 128 GB physical memory. This machine runs x86_64 Ubuntu Linux 10.04.2 LTS, kernel version 2.6.32-27. The 48 cores are provided by four AMD Opteron 6172 "Magny Cours" processors [Car, $\mathrm{CKD}^{+} 10$ ], each of which fits into a single G34 socket. Each processor contains two nodes, and each node has six cores. The 128 GB physical memory is provided by thirty-two 4 GB dual ranked RDIMMs, evenly distributed among four sets of eight sockets, with one set for each processor. As shown in Figure 8, these nodes, processors, and RAM chips form a hierarchy with significant differences in available memory bandwidth and number of hops required, depending upon the source processor core and the target physical memory location. Each 6 core node (die) has a dual-channel double data rate 3 (DDR3) memory configuration running at $1333 \mathrm{MHz}$ from its private memory controller to its own memory bank. There are two of these nodes in each processor package.

Bandwidth between each of the nodes and I/O devices is provided by four 16-bit HyperTransport 3 (HT3) ports, which can each be separated into two 8-bit HT3 links. Each 8-bit HT3 link has $6.4 \mathrm{~GB} / \mathrm{s}$ of bandwidth. The two nodes within a package are configured with a full 16-bit link and an extra 8-bit link connecting them. Three 8-bit links connect each node to the other three packages in this four package configuration. The remaining 16-bit link is used for I/O. Table 1 shows the bandwidth available between the different elements in the hierarchy.

Each core operates at $2.1 \mathrm{GHz}$ and has $64 \mathrm{~KB}$ each of instruction and data L1 cache and $512 \mathrm{~KB}$ of L2 cache. Each node has $6 \mathrm{MB}$ of L3 cache physically present, but, by default, $1 \mathrm{MB}$ is reserved to speed up cross-node cache probes.

\begin{tabular}{r|c|c} 
& AMD $(\mathrm{GB} / \mathrm{s})$ & Intel $(\mathrm{GB} / \mathrm{s})$ \\
\hline Local Memory & 21.3 & 17.1 \\
Node in same package & 19.2 & $n / a$ \\
Node on another package & 6.4 & 25.6
\end{tabular}

Table 1. Theoretical bandwidth available between a single node and the rest of the system.

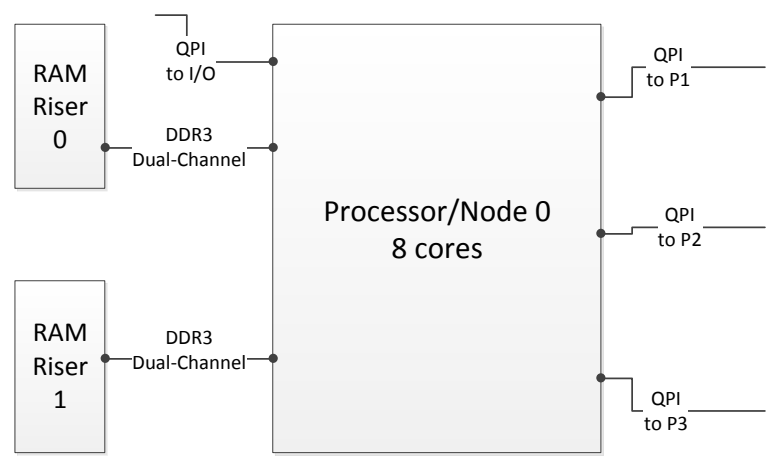

Figure 9. Interconnects for one processor in a quad Intel Xeon machine.

\section{A.2 Intel Hardware}

The Intel benchmark machine is a QSSC-S4R server with 32 cores and $256 \mathrm{~GB}$ physical memory. This machine runs x86_64 RedHat Enterprise Linux, kernel version 2.6.18194.11.4.el5. The 32 cores are provided by four Intel Xeon X7560 processors [Int, QSS]. Each processor contains 8 cores, which can be but are not configured to run with 2 simultaneous multithreads (SMT). As shown in Figure 9, these nodes, processors, and RAM chips form a hierarchy, but this hierarchy is more uniform than that of the AMD machine.

Each of the nodes is connected to two memory risers, each of which has a dual-channel DDR3 $1066 \mathrm{MHz}$ connection. The 4 nodes are fully connected by full-width Intel QuickPath Interconnect (QPI) links. Table 1 shows the bandwidth available between the different elements in the hierarchy.

Each core operates at $2.266 \mathrm{GHz}$ and $32 \mathrm{~KB}$ each of instruction and data $\mathrm{L} 1$ cache and $256 \mathrm{~KB}$ of L2 cache. Each node has $24 \mathrm{MB}$ of L3 cache physically present but, by default, $3 \mathrm{MB}$ is reserved to speed up both cross-node and cross-core caching. 